\title{
A retrospective descriptive investigation of adult patients receiving third-line antiretroviral therapy in the North West province, South Africa
}

\author{
Christian Rudman ${ }^{1}$, Michelle Viljoen ${ }^{2}$, Malie Rheeders ${ }^{1}$
}

1. Centre of Excellence for Pharmaceutical Services (PharmaCen), Division of Pharmacology, North-West University, Private Bag X6001, Potchefstroom 2520, South Africa.

2. Department of Pharmacology and Clinical Pharmacy, School of Pharmacy, Faculty of Natural Sciences, University of the Western Cape, Private Bag X17, Bellville 7535, South Africa.

\begin{abstract}
Background: Greater access and prolonged exposure to ART may inevitably lead to more treatment failure and increase the need for third-line ART (TLART) in a resource-limited setting.

Objective: To describe characteristics and resistance patterns of adult patients initiated on TLART in three districts of the North West province.

Method: All-inclusive retrospective descriptive investigation. Demographics and clinical variables were recorded from adult patient health records (2002-2017) and analysed.

Results: 21 Patients (17 females, 4 males) with median (IQR) age of 34 years (30.2-37.8) at HIV diagnosis and 45 years (39.5-47) at TLART initiation were included. Median duration (days) from HIV diagnosis to first-line ART initiation was 101 (37-367), treatment duration on first-line, second-line and between second-line failure and TLART initiation were: 1269 (765-2 343); 1512 (706-2096) and 71 (58-126) days respectively.

High-level resistance most prevalent were: nelfinavir/r $(85.7 \%)$, indinavir $/ \mathrm{r}(80.9 \%)$, lopinavir/r $(76.2 \%)$, emtricitabine and lamivudine (95.2\%), nevirapine (76.2\%) and efavirenz (71.4\%). Resistance to 3 major PI mutations in 95\% of patients and cross resistance were documented extensively.

Conclusion: This study support the need for earlier resistance testing. It firstly reported on time duration post diagnosis on various ART regimens and secondly resistance patterns of adults before TLART was initiated in these districts.

Keywords: HIV, Human immunodeficiency virus, third-line antiretroviral therapy, drug resistance patterns, salvage therapy. DOI: https://doi.org/10.4314/ahs.v20i2.2

Cite as: Rudman C, Viljoen M, Rheeders $M$. A retrospective descriptive investigation of adult patients receiving third-line antiretroviral therapy in the North West province, South Africa. Afri Health Sci. 2020; 20(2): 549-559. https: / / doi.org/ 10.4314/abs.v20i2.2
\end{abstract}

\section{Introduction}

Human immunodeficiency virus (HIV) infection remains a global pandemic, with an estimated 36.7 million people living with HIV globally at the end of $2016^{1,2}$. UNAIDS set out the ambitious 90-90-90 targets to be reached by end of 2020 , where $90 \%$ of people living with HIV should be diagnosed, $90 \%$ of the people diagnosed with HIV should receive antiretroviral therapy (ART) and $90 \%$ of those receiving ART should

\footnotetext{
Corresponding author:

Michelle Viljoen

Pharmacology and Clinical Pharmacy,

School of Pharmacy,

University of the Western Cape,

Private Bag X17, Bellville 7535, South Africa

Tel: +27219592641 Fax: +27 219593407

Email: mviljoen@uwc.ac.za
}

be virally suppressed to accomplish the eradication of the acquired immune deficiency syndrome (AIDS) by $2030^{3}$.

The number of people globally having access to ART was reported as 20.9 million in June $2017^{2}$. South Afri$\mathrm{ca}$, however, has the biggest epidemic in the world with an estimated 7.06 million HIV positive people in June 2017, which represents $12.6 \%$ of the total population and $18.0 \%$ of its adult population aged between 15 and 49 years ${ }^{4}$.

The South African National Department of Health (NDoH) implemented the first ART guidelines in 2004 through the Strategic Plan for HIV and AIDS and the operational plan for Comprehensive HIV and AIDS Management, Care and Treatment for South Africa ${ }^{5}$ as part of its public healthcare system and ART roll-out programme. This resulted in South Africa managing the largest ART programme in the world with 3.4 million people remaining on ART by the end of $2016^{6,7,8}$.

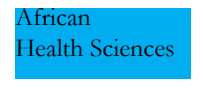

(C) 2020 Rudman C et al. Licensee African Health Sciences. This is an Open Access article distributed under the terms of the Creative commons Attribution License (https://creativecommons.org/licenses/BY/4.0), which permits unrestricted use, distribution, and reproduction in any medium, provided the original work is properly cited.

African Health Sciences Vol 20 Issue 2, June, 2020 
In 2013, the WHO emphasised that national policies should be developed for patients receiving third-line ART (TLART), as increased antiretroviral exposure and longer duration on ART would inevitably lead to more and more patients failing their first- and second-line ART due to drug resistance, increasing the need for more effective regimens for treatment-experienced patients $^{9,10,11}$.

The South African NDoH implemented TLART guidelines for patients in 2015 who did not achieve viral suppression on their respective PI-based second-line regimens for a minimum of 12 months after receiving intensified adherence counselling, to be eligible for genotypic antiretroviral resistance testing (GART). If resistance to a boosted PI was confirmed, the Adult Group of the Peer Review Committee for TLART from the NDoH would then consider the appropriate TLART for each individual patient ${ }^{12}$.

These TLART regimens are approximately five times more expensive than first-line ART ${ }^{13,14}$ and the continued increase in the number of patients on TLART will further place a burden on the already constrained national healthcare budget ${ }^{14}$. It is therefore paramount to effectively optimise patient management and care through rigorous policies and guidelines, especially in a resource-limited setting like South Africa.

The aim of this investigation was to describe and report on the clinical characteristics and HIV resistance patterns of all adult patients who were initiated on TLART in three districts of the North West Province in South Africa during the time of this investigation.

\section{Methods}

\section{Study design and population}

This study was an all-inclusive retrospective descriptive investigation of all adult patients approved for TLART. It was conducted in four main referral hospitals in three districts (Dr Kenneth Kaunda (DKK), Dr Ruth S. Mompati and Ngaka Modiri Molema) of the North West Province in South Africa.

Inclusion criteria for this study were all adult patients ( $\geq 18$ years) approved and already initiated on TLART by the end of April 2017. These adult patients should have been on a PI-based second-line ART regimen for at least 12 months, with confirmed boosted PI resistance by means of a GART performed by the National Health Laboratory Service (NHLS) of South Africa, before TLART could be initiated.

\section{Data collection and process}

Health records from TLART approved adult patients were first accessed by the responsible pharmacist at the respective referral hospitals and then the data were anonymously and retrospectively captured onto a specifically designed case report form by the researchers. The following demographical data were captured: date of birth and gender. Clinical markers (CD4 count and viral loads) for disease progression were captured at baseline (before any ART initiation, from 2002 onwards for this cohort) and at first- and second-line ART failure.

Time duration (in days) between HIV diagnosis and ARV initiation, duration on first-and second-line ART regimens and time spent between second-line failure and TLART initiation were recorded, including respective first- and second-line regimen combinations.

Genotypic antiretroviral test results, when patients experienced second-line ART failure, were captured for PIs, nucleoside reverse transcriptase inhibitors (NRTIs) and non-nucleoside reverse transcriptase inhibitor (NNRTIs) as susceptible, potential low-lev$\mathrm{el}$, intermediate and high level resistance. The NHLS incorporated the Stanford HIV Drug Resistance Database (HIVDB) genotyping tool to determine HIV-1 drug resistant mutations (http://hivdb.stanford.edu/).

\section{Ethical clearance}

Ethical approval was obtained from the Human Research and Ethics Committee of the North-West University (NWU-00340-16-A1), March 2017. As this was a retrospective health record investigation with no direct contact with any of the patients, no individual informed consent was obtained as this investigation would not have impacted on the treatment or care the patients would have received. No personal data was captured.

\section{Statistical analysis}

Descriptive statistics were calculated by means of IBM Statistical Package for the Social Sciences (SPSS $®)$, version 24. Median (interquartile range, IQR: 25th to 75th) was used for variables without a normal distribution and percentages (frequencies) were used for categorical variables.

\section{Results}

Twenty-four patients were approved and initiated on TLART by the end of April 2017; only 21 adult patients met the set inclusion criteria, with three patients 
younger than 18 years being excluded. Demographics and clinical characteristics of the patients are presented in Table 1. The median age at HIV diagnosis for this study population was 34 years (IQR: 30.2-36.8) and 45 years (IQR: 39.5-47) at TLART initiation. Although
HIV-1 subtype C is dominant in South Africa15, we were unable to record the specific HIV-1 subtype for this study population. The majority $(95 \%, 20 / 21)$ of these patients were diagnosed as HIV positive from 2004 onwards; one individual however was already diagnosed HIV positive in 2002.

Table 1: Demographics and clinical characteristics of patients $(n=21)$ initiated onto TLART

\begin{tabular}{|c|c|c|}
\hline \multicolumn{2}{|c|}{ Characteristics } & Median (IQR) \\
\hline \multicolumn{3}{|c|}{ Demographics } \\
\hline \multicolumn{2}{|c|}{ Age (years) at HIV diagnoses } & $34(30.3-36.8)$ \\
\hline \multicolumn{2}{|c|}{ Age (years) at TLART initiation } & $45(39.5-47)$ \\
\hline \multicolumn{2}{|c|}{ Female age (years) at TLART initiation $(n=17)$} & $45(38.5-47)$ \\
\hline \multicolumn{2}{|c|}{ Male age (years) at TLART initiation $(n=4)$} & $43.5(41.5-45.5)$ \\
\hline \multicolumn{3}{|l|}{ Clinical } \\
\hline \multicolumn{2}{|c|}{ CD4 count at baseline (cells $\left./ \mathrm{mm}^{3}\right)$} & $69(40.8-127.8)$ \\
\hline \multicolumn{2}{|c|}{ CD4 count at first-line failure $\left(\text { cells } / \mathrm{mm}^{3}\right)^{\mathrm{a}}$} & $79(26.5-124)$ \\
\hline \multicolumn{2}{|c|}{ CD4 count at second-line failure (cells/ $\mathrm{mm}^{3}$ ) } & $119(61.3-201.3)$ \\
\hline \multicolumn{2}{|c|}{ VL at baseline (copies/ml) } & $98000(45569-820000)$ \\
\hline \multicolumn{2}{|c|}{ VL at first-line failure $(\text { copies } / \mathrm{ml})^{a}$} & $100000(42085-248852)$ \\
\hline \multicolumn{2}{|c|}{$\mathrm{VL}$ at second-line failure (copies/ml) } & $73196(26210-197007)$ \\
\hline \multicolumn{3}{|c|}{ Durations (days) } \\
\hline \multicolumn{2}{|c|}{ Durations between HIV diagnoses and ART initiation } & $101(36.5-366.8)$ \\
\hline \multicolumn{2}{|c|}{ Duration on first-line ART ${ }^{c}$} & $1269(765-2343)$ \\
\hline \multicolumn{2}{|c|}{ Duration on second-line ART ${ }^{d}$} & $1512(706-2096)$ \\
\hline \multicolumn{2}{|c|}{ Duration between second-line failure and TLART initiation ${ }^{d}$} & $71(57.5-126)$ \\
\hline \multicolumn{3}{|c|}{ ART regimens } \\
\hline \multicolumn{3}{|c|}{ First-line regimens } \\
\hline \multicolumn{2}{|c|}{ 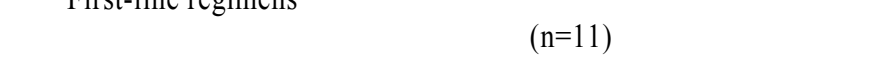 } & $3 \mathrm{TC} / \mathrm{d} 4 \mathrm{~T} / \mathrm{EFV}$ \\
\hline & $3 \mathrm{TC} / \mathrm{TDF} / \mathrm{EFV}$ \\
\hline \multirow{2}{*}{\multicolumn{2}{|c|}{$\begin{array}{l}(\mathrm{n}=1) \\
(\mathrm{n}=1)\end{array}$}} & 3TC/AZT/EFV \\
\hline & & FTC/TDF/EFV \\
\hline \multicolumn{3}{|c|}{ Second-line regimens ${ }^{d}$} \\
\hline & 3TC/AZT/LPV/r \\
\hline \multicolumn{2}{|c|}{$(\mathrm{n}=5)$} & 3TC/TDF/LPV/r \\
\hline & \\
\hline \multirow{3}{*}{\multicolumn{3}{|c|}{$\begin{array}{cc}(\mathrm{n}=2) & 3 \mathrm{TC} / \mathrm{ABC} / \mathrm{LPV} / \mathrm{r} \\
(\mathrm{n}=1) & 3 \mathrm{TC} / \mathrm{TDF} / \mathrm{AZT} / \mathrm{LPV} / \mathrm{r} \\
(\mathrm{n}=3) & \mathrm{ABC} / \mathrm{TDF} / \mathrm{LPV} / \mathrm{r} ; \mathrm{ddI} / \mathrm{TDF} / \mathrm{LPV} / \mathrm{r} \\
& 3 \mathrm{TC} / \mathrm{EFV} / \mathrm{LPV} / \mathrm{r}\end{array}$}} \\
\hline & & \\
\hline & & \\
\hline \multicolumn{3}{|c|}{ ARVs used in TLART } \\
\hline NRTI: & FTC $(n=15), 3$ TC $(n=6)$, TDF $(n=10), \operatorname{AZT~}(n=4)$ & $\mathrm{d} 4 \mathrm{~T}(\mathrm{n}=1)$ \\
\hline NNRTI: & $\operatorname{ETR}(n=4)$ & \\
\hline PI: & $\mathrm{DRV} / \mathrm{r}(\mathrm{n}=21)$ & \\
\hline INSTI: & RAL (n=9), DTG $(n=6)$ & \\
\hline $\begin{array}{l}\text { Median (IQR: } \\
{ }^{\mathrm{a}} \mathrm{n}=18, \text { three } \mathrm{p} \\
{ }^{\mathrm{b}} \mathrm{n}=18, \text { three } \mathrm{p} \\
{ }^{\mathrm{c}} \mathrm{n}=17, \text { three } \mathrm{p} \\
{ }^{\mathrm{d}} \mathrm{n}=20 \text {, one pat }\end{array}$ & $\begin{array}{l}\text { ot initiated onto recommended first-line ART regimens } \\
\text { ad missing data } \\
\text { ot initiated onto recommended first-line ART and one } \\
\text { missing data }\end{array}$ & ents had missing data \\
\hline
\end{tabular}

IQR, interguartile range; HIV, human immunodeficiency virus; TLART, third-line antiretroviral therapy; VL, viral load; ART, antiretroviral therapy; ARVs, antiretrovirals; 3TC, lamivudine; d4T, stavudine; EFV, efavirenz; TDF, tenofovir disoproxil fumarate; AZT, zidovudine; FTC, emtricitabine; LPV/r, lopinavir/ritonavir; ddl, didanosine; ABC, abacavir; ETR, etravirine; DRV/r darunavir/ritonavir; DTG, dolutegravir; RAL, raltegravir; NRTL, nucleoside/nucleotide reverse transcriptase inhibitor; NNRTI, non-nucleoside reverse transcriptase inhibitor; PI, protease inhibitor; INSTI, integrase strand transfer inhibitor

The median baseline CD4 count was 69 cells $/ \mathrm{mm}^{3}$ (IQR: 40.8-127.8), at first-line ART and second-line ART failure the CD4 counts were, 79 cells $/ \mathrm{mm}^{3}$ (IQR: 26.5-124) and 119 cells $/ \mathrm{mm}^{3}$ (IQR: 61.3-201.3).
The median baseline viral load (VL) was 98000 copies / $\mathrm{ml}$ (IQR: 45 569-820 000) and the VL at first-line and second-line ART failure were 100000 copies/ml (IQR: 42 085-248 852) and 73,196 copies/ml (IQR: 26210 $197007)$. 
Patients spent a median time of 101 days (IQR: 36.5366.8) between HIV diagnosis and ART initiation; 1 269 days (IQR: 765-2 343) on first-line ART; 1512 days (IQR: 706-2 096) on second-line ART and after failing their second-line ART regimen, patients waited a median additional 71 days (IQR: 57.5-126) for the approval and initiation of TLART.

Complete data on first-line ART regimens (Table 1) were only available for 17 patients. The combination regimen of lamivudine (3TC), stavudine (d4T) and efavirenz $(\mathrm{EFV})$ was most often initiated $(\mathrm{n}=11)$ followed by 3TC, tenofovir disoproxil fumarate (TDF) and EFV $(\mathrm{n}=4)$. One patient had missing data and three patients were initiated on ritonavir-boosted lopinavir (LPV/r) which is primarily reserved for second-line ART according to the adult guidelines in South Africa ${ }^{5,12,16}$. Complete data on PI-based ART regimens (Table 1) were available for 20 patients. The combination regimen of 3TC, zidovudine (AZT) and LPV/r was recorded most often $(n=7)$ followed by $3 \mathrm{TC}$, TDF and LPV $/ \mathrm{r}$ $(n=5)$.

The different combinations for TLART consisted of the following pharmacological classes and individual antiretroviral agents: NRTIs; emtricitabine (FTC), 3TC, TDF, AZT and d4T: NNRTI; etravirine (ETR): PI; darunavir/r (DRV/r): integrase strand transfer inhibitors (INSTIs); dolutegravir (DTG); raltegravir (RAL).

Figure 1 reflects the most prevalent $(>15 \%)$ viral mutations to PI's (major and minor), NRTIs and NNRTIs. Major mutations found against PI's with the highest prevalence $(n=20)$ were M46I $(n=15 ; 75 \%)$, V82A $(n=13 ; 65 \%)$ and I54V ( $n=13 ; 65 \%)$. The following major mutations were less prevalent $(5 \%$ respectively) and not reflected here: V82V, V82S, V82C, V32I, M46M, M46L, L90M, L76L, I50V, I50L, I47A.

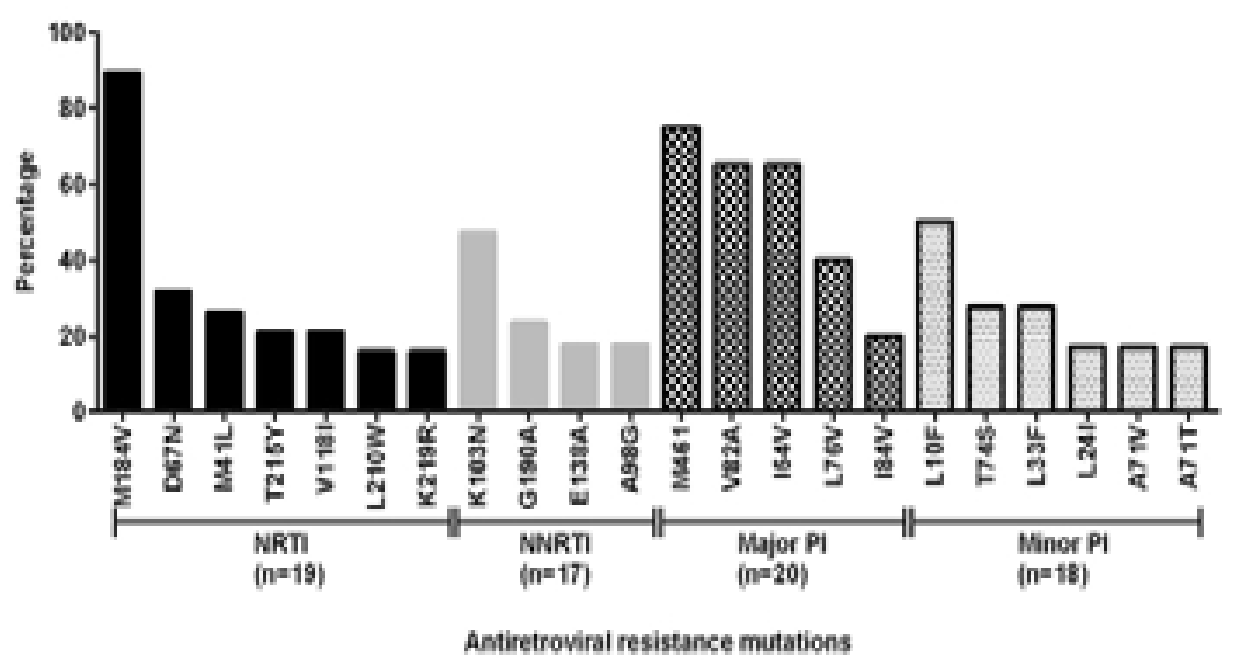

Figure 1: Percentage of most prevalent viral mutations to NRTIs, NNRTIs and PIs

$\mathrm{n}=$ number of patients who presented with viral resistant mutations to NRTIs, NNRTIs, major and minor PIs as respective classes. It does not account for individual mutations as patients had more than one mutation

The highest prevalence of minor viral mutations to PIs ( $\mathrm{n}=18$ ) were for $\mathrm{L} 10 \mathrm{~F}(\mathrm{n}=9 ; 50 \%)$, T74S and L33F $(\mathrm{n}=5 ; 27.7 \%)$. Less prominent minor mutations $(5.6 \%$ respectively) not reflected here were for L89V, L89M, L89I, L10V, L10L, K43T, K20I, F53L, F53F, A71I, and A71A.

Viral resistance mutations to the NRTI drug class with the highest prevalence $(n=19)$ were M184V $(n=17$; $89.5 \%), D 67 N(n=6 ; 31.6 \%), M 41 L(n=5 ; 26.3 \%)$ and T215Y and V118I ( $\mathrm{n}=4 ; 21.1 \%)$. The following mutations were less prevalent: V75I, T215F, Q151M, L74V, L74L, L74I, K70R, K70N, K65R, F77L, F116Y, D67D,
A62V (10.5\% respectively) and Y115F, V75M, T69T, T69S, T69D, M184M, K70E, K219Q, K219E, F77F, D67G (5.3\% respectively) not reflected in Figure 1.

Viral mutations identified within the NNRTI drug class with the highest prevalence $(n=17)$ were $K 103 \mathrm{~N}$ ( $n=8$; 47\%) and G190A ( $n=4 ; 23.5 \%)$. Mutations with lower prevalence not reflected in Figure 1 were: Y188L, V108I，P225H，K238N，K103R，K103K，K101S, $\mathrm{H} 221 \mathrm{Y}(11.8 \%$ respectively) and Y181C, V318F, V179V, V179I, V179D, V108V, V106M, V106I, N348N, N348I, K238S, K103S, K101R, K101P, K101H，K101E, H221H, F227L, E138G, E138E (5.9\% respectively). 
The total median number of PI resistance (major and minor) found per patient was three mutations (IQR: 2-3). The median number of major $(n=20) P I$ resistant mutations presented per patient was three (IQR: 3-4) and the median number of minor $(n=18)$ PI resistant mutations presented per patient was two (IQR: 2-3). The median number of NRTI resistant mutations pre- sented per patient was found to be three (IQR: 2-7) and the number of NNRTI resistant mutations presented per patient was three (IQR: 2-4).

The individual drug resistance was reported as low, intermediate, high and susceptible. Results on the level of resistance to the following respective pharmacological classes, are presented in Tables 2 (PIs), 3 (NRTIs) and 4 (NNRTIs) respectively.

Table 2: Percentage drug resistance reported for protease inhibitors (PIs)

\begin{tabular}{|l|c|c|c|c|}
\hline $\begin{array}{c}\text { Resistance level } \\
(\%)\end{array}$ & Low & Intermediate & High & Susceptible \\
\hline Atazanavir/r & 14.3 & 28.6 & 57.1 & 0 \\
\hline Darunavir/r & 19.1 & 33.3 & 0 & 47.6 \\
\hline Fosamprenavir/r & 19.1 & 14.3 & 66.7 & 0 \\
\hline Indinavir/r & 4.8 & 14.3 & 80.9 & 0 \\
\hline Lopinavir/r & 14.3 & 9.5 & 76.2 & 0 \\
\hline Nelfinavir/r & 0 & 14.3 & 85.7 & 0 \\
\hline Saquinavir/r & 19.1 & 57.1 & 19.1 & 4.8 \\
\hline Tipranavir/r & 33.3 & 38.1 & 4.8 & 23.8 \\
\hline
\end{tabular}

High-level resistance occurred for the following PIs: nelfinavir/r $(\mathrm{NFV} / \mathrm{r}) \quad(\mathrm{n}=18 ; \quad 85.7 \%), \quad$ indinavir $/ \mathrm{r}$ $(\mathrm{IDV} / \mathrm{r}) \quad(\mathrm{n}=17 ; \quad 80.9 \%), \quad \mathrm{LPV} / \mathrm{r} \quad(\mathrm{n}=16 ; \quad 76.2 \%)$, fosamprenavir/r $(\mathrm{FPV} / \mathrm{r})(\mathrm{n}=14 ; 66.7 \%)$ and atazanavir/r (ATV/r) ( $\mathrm{n}=12 ; 57.1 \%)$. None of the TLART patients indicated high-level resistance to darunvir/r $(\mathrm{DRV} / \mathrm{r})$ with 47.6\% reflecting susceptibility and all 21 patients received DRV/r as part of TLART.

Table 3: Percentage drug resistance for nucleoside/nucleotide reverse transcriptase inhibitors (NRTIs)

\begin{tabular}{|l|c|c|c|c|}
\hline $\begin{array}{c}\text { Resistance level } \\
(\%)\end{array}$ & Low & Intermediate & High & Susceptible \\
\hline Lamivudine & 4.8 & 0 & 95.2 & 0 \\
\hline Abacavir & 47.6 & 9.5 & 42.9 & 0 \\
\hline Zidovudine & 4.8 & 0 & 42.9 & 52.4 \\
\hline Stavudine & 9.5 & 0 & 42.9 & 47.6 \\
\hline Didanosine & 47.6 & 9.5 & 42.9 & 0 \\
\hline Emtricitabine & 4.8 & 0 & 95.2 & 0 \\
\hline Tenofovir & 9.5 & 14.6 & 28.6 & 47.6 \\
\hline
\end{tabular}

High-level resistance occurred for the following NRTIs: 3TC and FTC ( $\mathrm{n}=20 ; 95.2 \%$ respectively), ABC, AZT, d4T and ddI ( $\mathrm{n}=9 ; 42.9 \%$ respectively) and TDF $(n=6 ; 28.6 \%)$.
High-level resistance occurred for the following tested NNRTIs: nevirapine (NVP) $(\mathrm{n}=16 ; 76.2 \%)$ and EFV ( $n=15 ; 71.4 \%$ ) with susceptibility of $23.8 \%$ for etravirine and rilpivirine. Access to etravirine is limited in the public sector and rilpivirine is only available in private sector in South Africa. 
Table 4. Percentage drug resistance for non-nucleoside reverse transcriptase inhibitors (NNRTIs)

\begin{tabular}{|l|c|c|c|c|}
\hline $\begin{array}{c}\text { Resistance level } \\
(\%)\end{array}$ & Low & Intermediate & High & Susceptible \\
\hline Efavirenz & 4.8 & 14.3 & 71.4 & 9.5 \\
\hline Etravirine & 57.1 & 14.3 & 4.8 & 23.8 \\
\hline Nevirapine & 4.8 & 9.5 & 76.2 & 9.5 \\
\hline Rilpivirine & 38.1 & 19.1 & 19.1 & 23.8 \\
\hline
\end{tabular}

\section{Discussion}

The number of patients on TLART will increase as more and more patients develop resistance to second-line ART therapy ${ }^{10,11,17,18}$. The financial burden of TLART $^{13}$, which can be up to five times more expensive, on a resource-limited country is enormous and any intervention that can prevent this will be valuable.

This was the first study, to our knowledge, that described the characteristics and identified possible trends in the clinical profile of TLART patients in these three districts of the North West Province, taking into account certain clinical, demographical variables and resistance data. The majority $(81 \%)$ of this small study population were female which is not surprising as sub-Saharan woman have on average a $60 \%$ higher risk of being infected with $\mathrm{HIV}^{19}$.

Patients in our study population had a median age of 34 years at HIV diagnosis, similar to other much larger studies conducted in Soweto, South Africa during 2011, in United States of America (USA) during 2000-2009 and the INSIGHT START study ${ }^{20,21,22}$. The reproductive age range (15-49 years) receives more focus in research studies as this group is more prone to be sexually active and thus at greater risk of acquiring HIV infection $^{4,23}$, despite assumptions that HIV is less relevant in older generation ( $>49$ years) they should also be included in more studies ${ }^{23}$.

The median age of TLART initiated patients in our study population was 45 years, indicating a median time difference between HIV diagnoses and TLART initiation of at least 8.1 years. Published data on demographics of patients on TLART are limited, but a South African study reported a median age of 44 years when initiated onto salvage therapy and the median duration on ART prior to salvage was 8.9 years in a study conducted in the private sector ${ }^{24}$. These patients $(n=152)$ were enrolled in the Aid for AIDS (AfA) programme with salvage therapy being readily available from July 2007, contrary to the public sector rolling out TLART only from March $2013^{25}$.

This study's low median CD4 count of 69 cells $/ \mu \mathrm{L}$
(41-128) at baseline was similarly reported in studies in KwaZulu Natal (2011 to 2014) of 94 cells/ $\mu \mathrm{L}$ (2-384) and another large multicentre study (2000 to 2008) in South Africa which reported 96 cells/ $\mu \mathrm{L}$ (40-159) $)^{26,27}$. It is important to note that majority of baseline data for this descriptive investigation was recorded from 2004, when it was standard care for HIV positive patients to be first initiated onto co-trimoxazole prophylaxis treatment until they were eligible for ART with a CD4 count of $<200$ cells $/ \mu \mathrm{L}(2004-2010$ guidelines) and $<350$ cells/ $\mu \mathrm{L}$ (2013 guidelines) as recommended by the national ART guidelines of that time $e^{5,16,25}$. The low CD4 count at baseline and median 101 days (37-363) before first-line ART was initiated in this study population is evident from following these earlier guidelines.

Two randomised controlled trials (START and TEMPRANO ANRS 12136) addressed the optimal time to initiate ART and both studies indicated a significant clinical benefit from initiating ART in patients with a CD 4 count $>500$ cells $/ \mu \mathrm{L}$, rather than waiting for a patient`s CD4 count to reach a minimum threshold ${ }^{22,28}$. Further studies illustrated that in order to improve the lost to follow-up due to missed appointments by patients to initiate ART, patients can safely and effectively be initiated onto ART on the same day of HIV diagnosis $^{29,30}$ or within 14 days of ART eligibility ${ }^{31}$. The strategy to initiate ART irrespective of CD4 count or to "test and treat" has since been implemented in South Africa ${ }^{12,32}$ and recommended by the $\mathrm{WHO}^{33}$.

A large South African study conducted in nine HIV clinics between 2004 and 2013 identified age, gender, a constant low CD4 count during ART and appointments missed during the first six months (non-adherence) as the strongest predictors of first-line ART fail$u_{r}{ }^{34}$. In this study population, the median CD4 count remained very low at both first-line ART failure and at second-line ART failure (79 and 119 cells $/ \mu \mathrm{L}$ respectively) indicative of a consistently low CD4 count for the total median duration on ART. The lowest CD4 count reported in this study population (HIV diagnosis to TLART-initiation) was 3 cells $/ \mu \mathrm{L}$, although non adherence could not be reliably evaluated, it may be

African Health Sciences Vol 20 Issue 2, June, 2020 
possible that sustained low CD4 count could also have contributed to ART failure.

Taking into account the consistently low CD4 results, it is important to note the much higher median baseline VL of 98000 copies/ml (45 569-820 000) compared to the large cross sectional study in Soweto, South Africa, ( $\mathrm{n}=4$ 793) during 2011 with 13000 copies/ml (2 050-98 171) and higher CD4 count compared to this study population ${ }^{20}$.

In one of the largest studies ( $\mathrm{n}=19$ 645) from five public-sector facilities in South Africa where adults completed a minimum of 6 months on ART, the reported first-line failure at five years on treatment was $8-17 \%$ depended on the definition of confirmed virological failure $^{27}$. In this study the median VL at first-line failure was 100000 copies/ml (42 085-248 852). At second-line failure the median VL was 73196 copies/ml (26 210-97 007) for this study population which was similar to the private sector study ( $\mathrm{n}=152$ patients) with 82831 copies/ml (20 060-233 778) that were initiated on salvage therapy during 2007- 2011 at second-line ART failure ${ }^{24}$. Possible reasons for the high viral loads observed in this study at first-and second-line failure could be due to adherence issues (not monitored in this retrospective study) but also possibly long periods on failing regimens before ART was switched.

Major PI mutations are generally defined as "mutations that by themselves reduce the susceptibility to one or more drugs". Minor mutations are defined as having "little or no effect on susceptibility" and are sometimes also referred to as accessory, as they "reduce susceptibility only in combination with a major mutation or increase the replication fitness of viruses containing major drug resistance mutations" $" 35,36$.

Although up to $40 \%$ of adults in South Africa on PI-based second-line regimens experience virological failure ${ }^{37}$, all contributing risk factors have not been clearly accounted for. Due to the inclusion criteria of this study, a patient had to have documented resistance to a boosted PI after being treated with a PI-based second-line ART regimen for a minimum of 12 months before they could be considered for GART eligibility and TLART. It was therefore to be expected that 100\% of this study population experienced high-level resistance to $\geq 1 \mathrm{PI}$, and $95.23 \%$ had $\geq 1$ major PI mutation at the time GART was performed. The total median number of protease mutations (major and minor) found per patient in this study was 3 (IQR: 2-3) at the time of GART after spending a median 4.1 years on second-line treatment. Meintjes and co-workers reported that $75 \%$ of their AfA private setting $(n=152)$ cohort who qualified for salvage therapy with a median duration of 5.1 years on PI-based regimens presented with $\geq 3$ major protease mutations ${ }^{24}$. Protease inhibitor resistance mutations in a large public health study by van $\mathrm{Zyl}$ and co-workers (2013) reported that $11 \%$ of the patients on $\mathrm{LPV} / \mathrm{r}$ had $>1 \mathrm{LPV}$ resistant mutations but they did not report on the median duration of the LPV $/ \mathrm{r}$ treatment ${ }^{38}$.

It is important to consider that PI resistance requires consecutive accumulation of mutations with on-going exposure to a non-suppressive PI-based ART regimen ${ }^{39}$. The accumulation of protease mutations was closely linked to the time spent on a failing second-line ART regimens in studies conducted in Vietnam and Cambodia where different HIV-1 subtypes were relevant compared to South Africa ${ }^{40,41}$.

Adult patients failing second-line treatment for a duration of $0-12$ months and $>24$ months had median of $2(0-5)$ and $5(0-6)$ protease mutations (major and minor) per patient respectively, in a Nigerian cohort $(\mathrm{n}=61)$. They further estimated that 0.6 protease mutations developed for every six months they were maintained on a failing second-line regimen but reported that $38 \%$ of patients failing second-line ART did not have any protease mutations ${ }^{9}$. Chimbetete and co-workers reported that $51 \%$ of their participants $(\mathrm{n}=86)$ had major protease mutations, $14 \%$ and $12 \%$ had 3 or 2 PI mutations respectively at second-line ART failure with a median duration of 2.6 years on a PIbased regimen in Zimbabwe ${ }^{42}$. Contradictory to our study and the above mentioned studies no major resistance to PIs was reported in a study conducted in Gugulethu, a HIV referral centre in the Western Cape, with reported second-line ART failure in adult patients $(\mathrm{n}=43)$. These patients were on second-line treatment for a mean duration of 10 months and plasma samples were only taken after a mean period of 7 months post confirmed failure was first reported. They did however report that $91 \%$ of the individuals had minor protease mutations ${ }^{37}$. Another study at two clinics in Johannesburg $(n=75)$ reported that $39 \%$ of patients failing second-line regimen (LPV/ $\mathrm{r}$ and 2 NRTIs) did not have resistance mutations to protease or reverse transcriptase after spending an average of 16 months on second-line, suggesting that non-adherence could have contributed to the treatment failure ${ }^{18}$.

Other important risk factors that may lead to virological failure such as adherence should not be underestimated 
in the development of mutations and thus treatment failure. The Zimbabwean cohort study was conducted in patients with considered good adherence, they could therefore conclude that the regimen failure was due to resistance and not lack of adherence. They further established a significant association between age $(>24$ years, $\mathrm{p}=0.003$ ) and the development of major PI resistance and concluded that resistance was less prone in adolescents and young adults due to lack of adherence $^{42}$.

In this study, the total median duration on first- and second-line ART was median 42.3 and 50.4 months respectively. According to South Africa's national ART guidelines of 2015, a patient experiencing virological failure on a PI-based second-line ART regimen should be checked for tolerability and drug-drug interactions, and receive intensified adherence counselling before the VL can be repeated after 180 days $^{12}$. Taking into account the additional time prior to actual TLART initiation (median 71 days) and adding the guideline's recommended waiting time prior to VL repeat (180 days) could have resulted in approximately 251 days $(\approx 9$ months) spent on a failing second-line ART regimen before TLART was initiated. The importance of shortening the period between identifying second-line ART failure and initiating TLART is vital to minimise the number of protease mutations formed during prolonged time spent on failed second-line ART, was highlighted by various groups ${ }^{9,40,41,42}$. In this study all patients were older than 24 years and resistant to various PI's, neither adherence nor the HIV-1 subtype could be accounted, the median duration exposed to second-line was 50.4 months with approximately 9 months spent on failed second-line ART.

The three major PI mutations found in this study population with the highest prevalence were M46I (75\%), I54V and V82A (65\%). The major PI mutations present in the AfA private setting cohort who qualified for salvage therapy were in agreement with most of the major PI mutations found in this study population ${ }^{32}$. The major protease mutations most prevalent in $61 \mathrm{Ni}$ gerian patients failing their respective second-line ART between 2004 and 2011 were I54V, V82A, L10I and M46I ${ }^{9}$. Although results from the Gugulethu Clinic reported no major PI mutations they did however detect minor resistance mutations in $91 \%$ of their patients ${ }^{37}$. M46I is a nonpolymorphic mutation which is primarily selected by IDV, NFV, FPV, ATV and LPV and usually has a prevalence of $20 \%$ in treatment-experienced patients. M46I furthermore presents alone or in combina- tion with mutations V32I, I47V, L76V, I84V and L90M as was the case in this study as well. The prevalence of V82A and I54V mutations do not present in combination with M46I, they usually occur in combination with M46V, not present in this study population. Mutation V82A is a nonpolymorphic substrate-cleft mutation. It reduces the susceptibility to IDV and LPV and linked with cross-resistance to ATV and NFV. Lastly, mutation $154 \mathrm{~V}$ is also a nonpolymorphic mutation, largely selected by IDV and LPV and decreases the susceptibility of all the PI`s, except for DRV $/ \mathrm{r}^{36}$. This is also possibly why $100 \%$ of the patients in this cohort were initiated on DRV as part of their TLART, as this cohort had a $0 \%$ high level resistance, 19\% low-level resistance, 33\% intermediate resistance and 48\% susceptibility to DRV.

The emergence of PI cross-resistance mutations also played a role in this study, with the highest level of resistance against NFV (86\%), IDV (81\%), FPV (67\%) and ATV (57\%) even though these patients were only treatment-experienced on $\mathrm{LPV} / \mathrm{r}$. This observation of cross resistance development to other PI's has been well documented previously ${ }^{38,41}$. PI`s share similar chemical structures, therefore cross-resistance is often observed and clarifies the observation of patients experiencing high-level resistance to PI`s they were never previously been exposed to ${ }^{43}$.

Considering the latter, we acknowledge that all the mutations reflected in figure 1 synergistically and holistically affected the level of resistance found in this study population, but the clinical effects of the mutations, as described in the Stanford University HIV Drug Resistance Database, can be seen in the data of this study population and is a valuable contribution to the resistance mutation data on TLART patients in South Africa.

Resistance mutations for the NRTI's with the highest prevalence were M184V (89.5\%) followed by the thymidine analogue mutations (TAM's), D67N (31.6\%) and M41L (26.3\%). These frequencies corresponded with recent results from the Zimbabwean cohort who reported $67 \%$ prevalence for $\mathrm{M} 184 \mathrm{~V}$ and $36 \%$ for $\mathrm{D} 67 \mathrm{~N}^{42}$ and a Nigerian cohort with $66 \%$ for M184V, $43 \%$ for $\mathrm{M} 41 \mathrm{~L}$ and $34 \%$ for $\mathrm{D} 67 \mathrm{~N}^{9}$ in patients who failed second-line ART during 2004-2011. M184V is the most prominent NRTI mutation in Africa and mainly affects resistance to 3TC and FTC as was also shown in this study and others ${ }^{42,43,44}$.

The M184V mutation is clinically significant as it is primarily selected by 3TC and FTC and reduces the susceptibility of both these drugs by $>100$ fold, but in 
contrast causes an increase in the susceptibility to AZT, $\mathrm{d} 4 \mathrm{~T}$ and TDF and decreases the rate at which $\mathrm{AZT}$, $\mathrm{d} 4 \mathrm{~T}$ and TDF resistance occurs ${ }^{36}$. This high prevalence $(89.5 \%)$ of the M184V mutation found in this study population can possibly be attributed to the number of patients who were initiated on a 3TC based first-line (76\%) and second-line ART regimen (71\%). It is evident that this $\mathrm{M} 184 \mathrm{~V}$ mutation had a significant effect on 3TC and F'TC resulting in 95\% high-level resistance, but M184V may have contributed to the increased susceptibility to AZT (52\%), d4T and TDF (48\%) although other factors may have had an impact. The TAM's, in this case $\mathrm{D} 67 \mathrm{~N}$ and $\mathrm{M} 41 \mathrm{~L}$, are known to accumulate in patients who remain on a failing ART regimen ${ }^{45,46}$. Unfortunately GART results were not available at firstline failure for this study cohort. If these mutations were thus already present when switched to second-line ART they could have negatively impacted the second-line treatment ${ }^{45,46}$ notwithstanding the long duration on failed second-line therapy in this small cohort.

High-level resistance to NNRTI's were found for NVP $(76.2 \%)$ and $\mathrm{EFV}(71.4 \%)$ and mutations most prevalent for NNRTI's were K103N (47\%) and G190A $(23.5 \%)$. Mutation $\mathrm{K} 103 \mathrm{~N}$ is a nonpolymorphic mutation that primarily selects for NVP and EFV and reduces the susceptibility of these drugs by fifty- and twentyfold respectively. Mutation G190A is also a nonpolymorphic mutation that primarily selects for NVP and $\mathrm{EFV}^{36}$. Major NNRTI mutations found in the Nigerian cohort ${ }^{9}$, for patients failing second-line ART between 2004-2011, were K103N, Y181C (26\%) and G190A (16\%). The Y181C mutation was much less prevalent in this study at $5.9 \%$.

It was not clear why three patients were initiated on regimens containing $\mathrm{LPV} / \mathrm{r}$, primarily reserved for second-line ART in adults in South Africa since $2004^{6,13,14,15,16,24}$. Reasons such as drug intolerability, drug-drug interactions, missing health information or not accurately recorded dates and possible errors in following the national ART guidelines may have played a role.

This study had several limitations that need to be taken into consideration. Adherence to ART could not be accurately investigated retrospectively and although this was an all-inclusive sample, only a small number of patients were initiated on TLART in the North West Province during this investigational period. Baseline GART testing did not form part of the South African guidelines and thus prior resistance data was not available until patients were considered for TLART. Due to the retrospective nature of this study, incomplete patient health records could have contributed to vital missing information.

\section{Conclusion}

The increase in the number of TLART patients in future will place a significant financial and infrastructural burden on South Africa. This small descriptive study contributed and improved the knowledge on the clinical characteristics and resistance patterns of TLART patients in order to improve and optimise HIV management and care of these patients living in a resource limited setting, such as the North West Province, in South Africa.

The major NRTI mutations identified were M184V, D67N and M41L, major NNRTI mutations were K103N and G190A and the major PI mutations were M46I, V82A and I54V. The large extent of PI cross-resistance found amongst these patients predominantly on LPV/r was described previously but it highlights the importance of more frequent and earlier GART testing, although costly, as patients spent longer durations on failing ART regimens. This will enable the prescriber to choose maximum suppressive ART combinations to be used as soon as possible.

This is the first study, to our knowledge, reporting on the different time duration post diagnosis, on various ART regimens and resistance patterns of adults on TLART in these districts. It highlights the median 71 days between documented second-line ART failure and the actual initiation of TLART and the enormous burden this system is already facing in the relatively early phases of TLART initiation.

\section{Acknowledgements}

Data analysis was performed in collaboration with Marike Cockeran at the School for Computer, Statistical and Mathematical Sciences, Faculty of Natural Sciences, North-West University, Potchefstroom, South Africa.

\section{Conflict of interest}

The authors declare no competing interests.

\section{Author contributions}

MV conceptualised the study and received ethics clearance; CR collected data for analysis; CR and MR conducted data analysis. Interpretation of data was conducted by all the authors. CR drafted the first version of the manuscript, which was finalised with significant contributions from both MR and MV. The final manuscript was approved by all authors. 


\section{References}

1. UNAIDS: Global AIDS update. 2016. Retrieved November 2, 2017 from http://www.unaids.org/sites/ default/files/media_asset/global-AIDS-update-2016_ en.pdf

2. UNAIDS: Fact Sheet - World Aids Day. 2017. Retrieved November 2, 2017 from http://www.unaids. $\mathrm{org} / \mathrm{sites} /$ default/files/media_asset/UNAIDS_FactSheet_en.pdf.

3. UNAIDS: 90-90-90 An ambitious treatment target to help end the AIDS epidemic. 2014. Retrieved June 17, 2018 from http://www.unaids.org/sites/default/files/ media_asset/90-90-90_en.pdf.

4. StatsSA: Mid-year population estimates. StatsSA; 2017.

Retrieved November 2, 2017 from http:/ /www.statssa. gov.za/publications/P0302/P03022017.pdf.

5. National Department of Health, Republic of South Africa. National Antiretroviral Treatment Guidelines. 2004. Retrieved November 2, 2017 from http:// apps.who.int/medicinedocs/documents/s17758en/ s17758en.pdf.

6. National Department of Health, Republic of South Africa. Annual report 2014/2015. 2015. Retrieved November 2, 2017 from http://www.health-e. org.za/wp-content/uploads/2015/10/Department-of-Health-Annual-Report-201415.pdf.

7. National Department of Health, Republic of South Africa. Annual report 2015/16. 2016. Retrieved November 2, 2017 from http://www.health.gov.za/index. php/2014-08-15-12-56-31?.

8. South Africa National Aids Council (SANAC). South Africa global AIDS response progress report (GARPR). 2015. Retrieved November 2, 2017 from http:/ / sanac.org.za/wp-content/uploads/2016/06/GARPR_ report-high-res-for-print-June-15-2016.pdf.

9. Rawizza HE, Chaplin B, Meloni ST, et al. Accumulation of protease mutations among patients failing second-line antiretroviral therapy and response to salvage therapy in Nigeria. PLoS One. 2013; 8:e73582.

10. Magambo B, Nazziwa J, Bbosa N, et al. The arrival of untreatable multidrug-resistant HIV-1 in sub-Saharan Africa. AIDS. 2014; 28(9):1373-74.

11. World Health Organisation. Consolidated guideline on the use of antiretroviral drugs for treating and preventing HIV infection. First edition 2013. Retrieved November 2, 2017 from http://apps.who.int/iris/bitst ream $/ 10665 / 85321 / 1 / 9789241505727$ eng.pdf?ua=1. 12. National Department of Health, Republic of South Africa. National consolidated guidelines for the prevention of mother-to-child transmission of HIV
(PMTCT) and the management of HIV in children, adolescents and adults. 2015. Retrieved November 2, 2017 from https://www.health-e.org.za/2015/07/02/ guidelines-national-consolidated-guidelines-for-pmtctand-the-management-of-hiv-in-children-adolescentsand-adults/.

13. Meyer-Rath G, Pillay Y, Blecher M, et al. Total cost and potential cost savings of the national antiretroviral treatment (ART) programme in South Africa 2010 to 2017. Proceedings of XVIII International AIDS Conference; Durban. 2010.

14. Onoya D, Nattey C, Budgell E, et al. Predicting the need for third-line antiretroviral therapy by identifying patients at high risk for failing second-line antiretroviral therapy in South Africa. AIDS Patient Care STDs. 2017; 31:205-12.

15. Wilkinson E, Rasmussen D, Ratmann O, Stadler T, Engelbrecht S, de Oliveira T. Origin, imports and exports of HIV-1 subtype $C$ in South Africa: a historical perspective. Infect Genet Evol. 2016; 46:200-8. doi:10.1016/j.meegid.2016.07.008

16. National Department of Health, Republic of South Africa. The South African antiretroviral treatment guidelines South Africa. 2010. Retrieved November 2, 2017 from http://apps.who.int/medicinedocs/documents/s19153en/s19153en.pdf.

17. Country factsheet South Africa 2016. Retrieved November 2, 2017 from www.unaids.org/en/regionscountries/countries/southafrica.

18. Wallis CL, Mellors JW, Venter WDE, Sanne I, Stevens W. Protease inhibitor resistance is uncommon in HIV-1 subtype $\mathrm{C}$ infected pateints on failing second-line lopinavir/ $\mathrm{r}$-containing antiretroviral therapy in South Africa. AIDS Res Treat. 2011; 2011:769627. doi:10.1155/2011/769627

19. Magadi MA. Understanding the gender disparity in HIV infection across countries in sub-Saharan Africa: evidence from the Demographic and Health Surveys. Sociology of Health and Illness. 2011; 33(4): 522-39.

20. Govender S, Otwombe K, Essien T, et al. CD4 counts and viral loads of newly diagnosed HIV-infected individuals: implications for treatment as prevention. PloS One. 2014; 9:e90754.

21. Buchacz K, Armon C, Palella FJ, et al. CD4 cell counts at HIV diagnosis among HIV outpatient study participants, 2000-2009. AIDS Res Treat. 2012; 2012, ID 869841. doi:10.1155/2012/869841

22. INSIGHT START Study Group. Initiation of antiretroviral therapy in early asymptomatic HIV Infection. N Engl J Med. 2015; 373:795-807. doi:10.1056/ NEJMoa1506816 
23. Freeman E, Anglewicz P. HIV prevalence and sexual behaviour at older ages in rural Malawi. Int J STD AIDS. 2012; 23(7): 490-96. doi:10.1258/ijsa.2011.011340

24. Meintjes G, Dunn L, Coetsee M, et al. Third-line antiretroviral therapy in Africa: effectiveness in a Southern African retrospective cohort study. AIDS Res Ther. 2015; 12:39. doi:10.1186/s12981-015-0081-8

25. National Department of Health, Republic of South Africa. The South African antiretroviral treatment guidelines. 2013. Retrieved November 2, 2017 from http://www.kznhealth.gov.za/medicine/2013_art_ guidelines.pdf.

26. Narainsamy D, Mahomed S. Delays in switching patients onto second-line antiretroviral treatment at a public hospital in eThekwini, Kwazulu-Natal. South Afr J HIV Med. 2017; 18(1):a696. doi.org/10.4102/sajhivmed.v18i1.696

27. Fox MP, Van Cutsem G, Giddy J, et al. Rates and predictors of failure of first-line antiretroviral therapy and switch to second-line ART in South Africa. J Acquir Immune Defic Syndr. 2012; 60:428-37.

28. TEMPRANO ANRS 12136 Study Group. A trial of early antiretrovirals and isoniazid preventive therapy in Africa. N Eng J Med. 2015; 2015:808-22. doi:10.1056/ NEJMoa1507198

29. Rosen S, Maskew M, Fox MP, et al. Initiating antiretroviral therapy for HIV at a patient's first clinic visit: the RapIT randomized controlled trial. PLoS Med. 2016; 13:e1002015.

30. Pilcher CD, Ospina-Norvell C, Dasgupta A, et al. The effect of same-day observed initiation of antiretroviral therapy on HIV viral load and treatment outcomes in a US public health setting. I Acquir Immune Defic Syndr. 2017; 74:44-51.

31. Amanyire G, Semitala FC, Namusobya J, et al. Effects of a multicomponent intervention to streamline initiation of antiretroviral therapy in Africa: a steppedwedge cluster-randomised trial. The Lancet HIV. 2016; 3:e539-e548.

32. Meintjes G, Moorhouse MA, Carmona S, et al. Adult antiretroviral therapy guidelines 2017. South Afr J HIV Med. 2017; 18:1-24.

33. World Health Organisation. Guideline on when to start antiretroviral therapy and on pre-exposure prophylaxis for HIV. 2015. Retrieved November 2, 2017 from http://apps.who.int/iris/bitstre am/10665/186275/1/9789241509565_eng.pdf.

34. Rohr JK, Ive P, Horsburgh CR, et al. Developing a predictive risk model for first-line antiretroviral therapy failure in South Africa. I Int AIDS Soc. 2016; 19(1):20987. doi:10.7448/IAS.19.1.20987

35. Wensing AM, Calvez V, Günthard HF, et al. Update of the drug resistance mutations in HIV-1. Top Antivir Med. 2015; 23(4):132-41.

36. Stanford University HIV Drug Resistance Database. Major HIV-1 drug resistance mutations. 2017. Retrieved June 12, 2018 from https://hivdb.stanford.edu/ updated 11 Dec 2017.

37. Levison JH, Orrell, C, Gallien S, et al. Virologic failure of protease inhibitor-based second-line antiretroviral therapy without resistance in a large HIV treatment program in South Africa. PLoS One. 2012; 7(3) e32144. 38. Van Zyl GU, Liu TF, Claassen M, et al. Trends in genotypic HIV-1 antiretroviral resistance between 2006 and 2012 in South African patients receiding first-and second-line antiretroviral treatment regimens. PLoS One. 2013; 8(6):e67188.

39. Condra J. Resistance to HIV protease inhibitors. Haemophilia. 1998; 4:610-615.

40. Thao, VP, Quang VM, Day JN, et al. High prevalence of PI resistance in patients failing second-line ART in Vietnam. J Antimicrob Chemother. 2016; 71(3): 762-74. doi:10.1093/jac/dkv385

41. Nerrienet E, Nouhin J, Ngin S, et al. HIV-1 Protease inhibitors resistance profiles in patients with virological failure on LPV/r-based 2nd line regimen in Cambodia. J AIDS Clinic Res. 2012; S5:003. doi.org/10 4172/21556113 S5-003

42. Chimbetete C, Katzenstein D, Shamu T, et al. HIV1 drug resistance and third-line therapy outcomes in patients failing second-line therapy in Zimbabwe. Open Forum Infect Dis. 2018; 5(2):ofy005. doi:10.1093/ofid/ ofy005.

43. Arts EJ, Hazuda DJ. HIV-1 antiretroviral drug therapy. Cold Spring Harb Perspect Med. 2012; 2:a007161.

44. Ssemwanga D, Lihana RW, Ugoji C, et al. Update on HIV-1 acquired and transmitted drug resistance in Africa. AIDS Review. 2015; 17(1):3-20.

45. Cozzi-Lepri A, Phillips AN, Martinex-Picado J. Rate of accumulation of thymidine analogue mutations in patients continuing to receive virologically failing regimens containing zidovudine or stavudine: implications for antiretroviral therapy programs in resource-limited settings. J Infect Dis. 2009; 200(5): 687-97.

46. Sigaloff KCE, Ramatsebe T, de Wit VR, Wallis CL, Stevens WS. Accumulation of HIV drug resistance mutations in patients failing first-line antiretroviral treatment in South Africa. AIDS Res Hum Retro. 2011; 28(2):171-175. doi:10.1089/aid.2011.0136 